\title{
Third-harmonic Mie scattering from semiconductor nanohelices
}

\author{
Lukas Ohnoutek ${ }^{1,2,8}$, Ji-Young Kim ${ }^{3,4,8}$, Jun Lu ${ }^{3,4}$, Ben J. Olohan ${ }^{1,2}$, Dora M. Răsădean ${ }^{5}$, \\ G. Dan Pantoș ${ }^{5}$, Nicholas A. Kotov ${ }^{3,4} \bowtie$ and Ventsislav K. Valev $\mathbb{1}^{1,2,6,7 凶}$
}

\begin{abstract}
Chiroptical spectroscopies provide structural analyses of molecules and nanoparticles but they require sample volumes that are incompatible with generating large chemical libraries. New optical tools are needed to characterize chirality for the ultrasmall $(<1 \mu \mathrm{l})$ volumes required in the high-throughput synthetic and analytical stations for chiral compounds. Here we show experimentally a novel photonic effect that enables such capabilities-third-harmonic Mie scattering optical activity-observed from suspensions of $\mathrm{CdTe}$ nanostructured helices in volumes $<<1 \mu \mathrm{l}$. Third-harmonic Mie scattering was recorded on illuminating CdTe helices with 1,065, 1,095 and 1,125 nm laser beams and the intensity was around ten-times higher in the forward direction than sideways. The third-harmonic ellipticity was as high as $3^{\circ}$ and we attribute this effect to the interference of chiral and achiral effective nonlinear susceptibility tensor components. Third-harmonic Mie scattering on semiconductor helices opens a path for rapid high-throughput chiroptical characterization of sample volumes as small as $10^{-5} \mu$.
\end{abstract}

C ombinatorial nanochemistry allows tens to millions of mixtures to be produced in a single process ${ }^{1,2}$, generating large chemical libraries ${ }^{3-7}$ and making implementation of artificial intelligence algorithms possible. Following the rapid development of chiral nanostructures ${ }^{8-13}$, such synthetic and analytical platforms can be applied to the high-throughput assessment of enzyme mimics, contrast agents, antibiotic agents, drug delivery vehicles, as well as other applications of these bioinspired materials. These analyses should be carried out in microplates of $1,536,3,456$ or 9,600 wells, with sample volumes as small as, and smaller than, $1 \mu$ (ref. ${ }^{14}$ ), but the traditional methods of chiral optical (chiroptical) spectroscopy typically require volumes that are 100-1,000 times larger, highlighting the dire need for the development of new optical tools for performing rapid characterization of chiral nanocompounds in ultrasmall volumes. Nonlinear chiroptical effects can potentially be observed in focal volumes down to tens of micrometres and are therefore well suited to microplates with even the highest number of wells. Although nonlinear chiroptical effects applicable to isotropic liquids have been reported ${ }^{15-20}$, they are technologically too complex for the needs of combinatorial nanochemistry and high-throughput synthesis. For instance, in the case of hyper Rayleigh scattering optical activity, the geometry of the well plates is not convenient for registration of the nonlinear Rayleigh scattering. A $4 \pi$ integration sphere with the individual sample in the centre would be typically required to reduce artefacts and cross-talk between adjacent samples.

Nonlinear chiroptical characterization of inorganic nanostructures in small volumes may be possible via circularly polarized Mie scattering spectroscopy. This scattering modality is advantageous over Rayleigh scattering as Mie scattering occurs within a small solid angle, which simplifies the registration and polarization analysis of the scattered photons, and is compatible with the requirements of a microplate geometry. Rayleigh and Mie scattering were extensively investigated in metallic achiral particles ${ }^{21,22}$, but not so much for chiral particles ${ }^{23}$, whose interactions with circularly polarized photons are more complex and therefore more informative than for achiral scatterers. For instance, giant chiral optical effects have been reported in chiral plasmonic metamaterials ${ }^{24}$ and metasurfaces ${ }^{25}$. Moreover, Mie scattering from chiral metallic particles and their assemblies can lead to exceptionally strong dependence on light polarization, torque transfer and spin-polarized electron ejection $^{26,27}$. Despite the broad interest that chiral non-metallic particles hold in the context of ultrafast and miniaturized devices ${ }^{28-30}$, their Mie scattering properties are even less known ${ }^{31}$. Light-matter interactions with non-metallic particles may include electric and magnetic dipoles and multipoles ${ }^{32,33}$, as well as profit from Fano ${ }^{34}$ and anapole resonances ${ }^{35}$. Compared with plasmonic counterparts, the electric and magnetic contributions in these non-metallic nanoparticles may become comparable ${ }^{36}$, which leads to enhanced chiroptical properties. The nonlinear chiroptical properties of such individual particles are currently unknown.

Here we investigate the nonlinear scattering processes of $\mathrm{CdTe}$ semiconductor helices ${ }^{37}$ and we report a new chiroptical effect. These nanostructured helices are self-assembled from CdTe nanoparticles. They have a length of 5-8 $\mu \mathrm{m}$, suitable for Mie scattering. For brevity, they will be referred to as nanohelices, not only because they display some nanoscale dimensions (for example, thickness $\approx 25 \mathrm{~nm}$ ), but also because they retain to a large degree the nanoscale dimensions of individual self-assembled nanowires braided together ${ }^{37}$. The semiconductor nanohelices were obtained at $99.5 \%$ enantiomeric purity with both left $(\Lambda)$ and right $(\Delta)$ handedness. They are randomly dispersed within an isotropic liquid environment in all the samples in this study. Their dispersions reveal strong chiroptical third-harmonic Mie scattering (THMS) that was observed for three different wavelengths in the near-infrared range of the electromagnetic spectrum. Their THMS is characterized by around ten-times

${ }^{1}$ Centre for Photonics and Photonic Materials, University of Bath, Bath, UK. ${ }^{2}$ Centre for Nanoscience and Nanotechnology, University of Bath, Bath, UK. ${ }^{3}$ Department of Chemical Engineering, University of Michigan, Ann Arbor, MI, USA. ${ }^{4}$ Biointerfaces Institute, University of Michigan, Ann Arbor, MI, USA. ${ }^{5}$ Department of Chemistry, University of Bath, Bath, UK. ${ }^{6}$ Centre for Therapeutic Innovation, University of Bath, Bath, UK. ${ }^{7}$ Department of Basic and Applied Sciences for Engineering, the Sapienza University of Rome, Rome, Italy. ${ }^{8}$ These authors contributed equally: Lukas Ohnoutek, Ji-Young Kim. 凶e-mail: kotov@umich.edu; v.k.valev@bath.ac.uk 
a
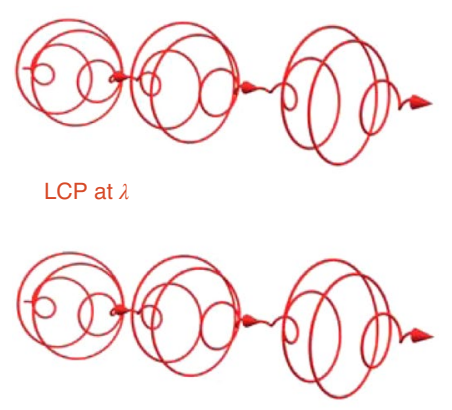
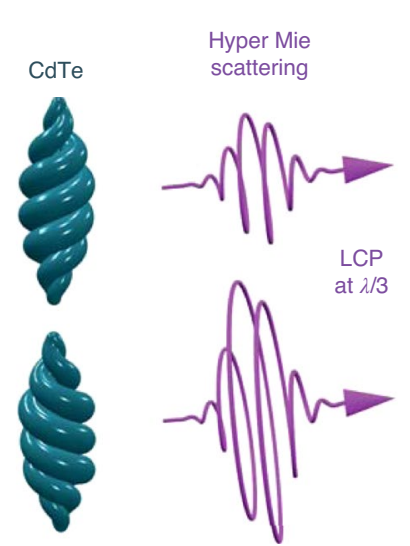

b

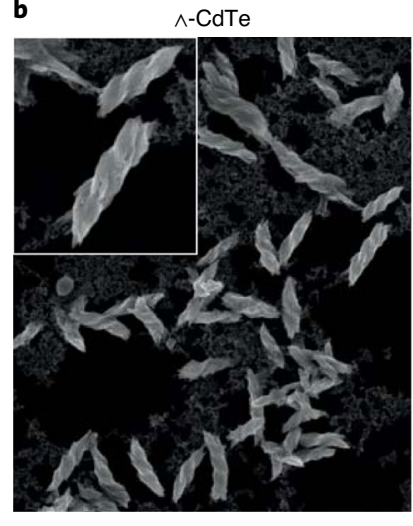

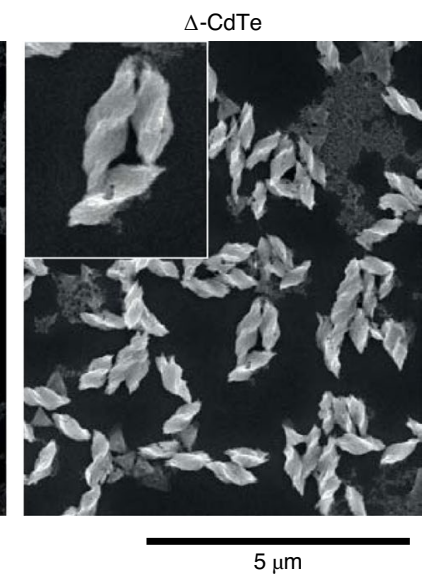

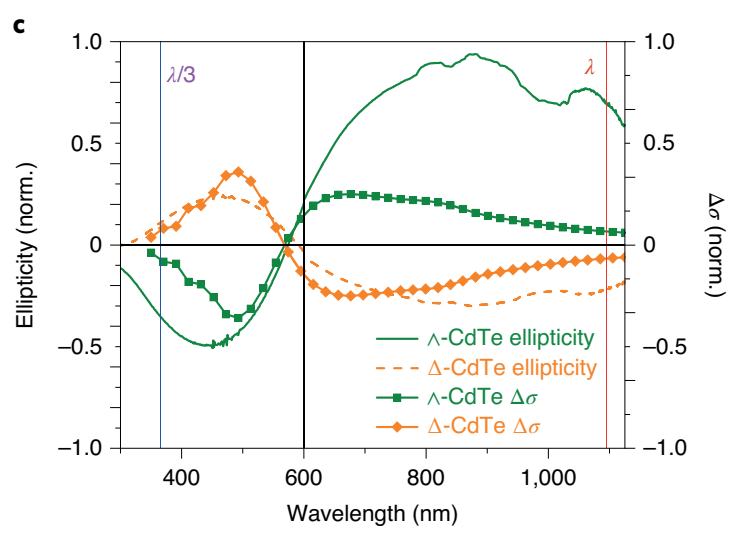

d

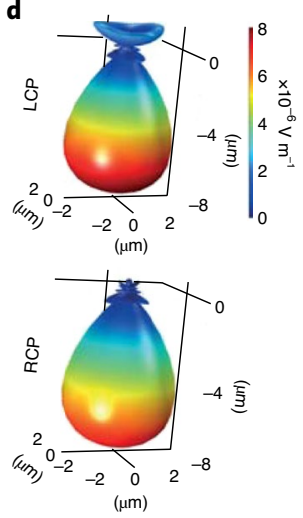

e

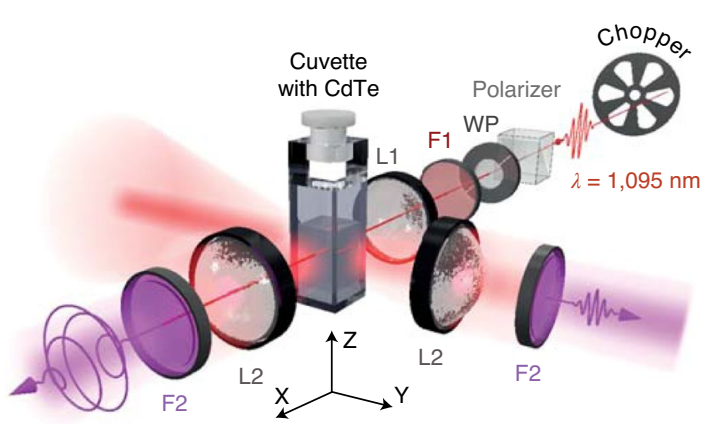

Fig. 1 | CdTe nanohelices for third-harmonic Mie scattering optical activity. a, Schematic diagram of THMS optical activity. Following illumination with LCP light at wavelength $\lambda$, the intensity of THMS light (at $\lambda / 3$ ) depends on the handedness of the CdTe nanohelices. $\mathbf{b}$, Scanning electron micrographs of the left- and right-handed CdTe nanohelices, indicated as $\Lambda$-CdTe and $\Delta$-CdTe, respectively. c, Experimental ellipticity spectra and numerically computed differential extinction cross-section spectra from the nanohelices, in the linear optical regime. The wavelengths of illumination and detection for the THMS experiments are indicated with coloured vertical lines. The black vertical line separates data obtained using different detectors. $\mathbf{d}$, Emission patterns of a $\Lambda$-CdTe nanohelix illuminated along the main axis for LCP and RCP light. e, A diagram of the TH scattering set-up. WP, quarter-wave plate; F1, optical filter allows $\lambda$; L1, focusing lens; L2, collimating lens; F2, band-pass filter that allows only $\lambda / 3$ through.

stronger third-harmonic (TH) emission in the forward direction than at $90^{\circ}$ angle with respect to the incident light. The nonlinear ellipticity can be as high as $3^{\circ}$ and it changes sign for $\Lambda$ - and $\Delta$-CdTe nanohelices. The chiroptical contrast reverses between forward and $90^{\circ}$ angle emission, which reveals the phase relationship between chiral and achiral effective tensor components of the TH susceptibility. Furthermore, the chiroptical effect can be observed both in the forward emitted circularly polarized TH light and in the linearly polarized TH light emitted sideways. Finally, we demonstrate a successful chiroptical characterization of CdTe nanohelices in a minuscule $(<1 \mu \mathrm{l})$ volume using THMS.

\section{Results}

Third-harmonic Mie scattering optical activity occurs when three circularly polarized photons at the fundamental frequency produce a single photon at the triple frequency, with a conversion efficiency that depends on the chirality of the CdTe helix and the handedness of the incident circularly polarized light (Fig. 1a). This effect is a manifestation of optical activity in the general sense of the term, that is, where it designates any change of the intensity or polarization of light that is due to material chirality. Third-harmonic Mie scattering optical activity was never observed before for any semiconductor, metal, dielectric or biological samples.

CdTe nanohelices were synthesized via a method used by Yan et al. ${ }^{37}$ to produce helicoids with a geometry approaching that of twisted ribbons (Fig. 1b). On analysing the SEM microscopy images and geometrical parameters of the nanohelices (in one batch: thickness, $140 \pm 20 \mathrm{~nm}$; width, $453 \pm 70 \mathrm{~nm}$; pitch, $302 \pm 45 \mathrm{~nm}$ ), one can notice that their uniformity originates from the self-limited assembly of the nanoparticles controlled by electrostatic repulsion ${ }^{38}$. Circular dichroism spectra of the $\Lambda$ - and $\Delta$-nanohelices have similar bisignate shapes but opposite polarities. The differential extinction cross-section spectra of the three-dimensional helix model (Supplementary Fig. 1), calculated using the finite-difference time domain method, match well with the experimental circular dichroism spectra (Fig. 1c). The difference in amplitude of experimental circular dichroism peaks is attributable to a difference in concentration of the prepared suspensions. Figure 1d shows the scattering pattern of a $\Lambda$-CdTe nanohelix at a wavelength identical to that of the incident beam, illuminated along the main axis, for left circularly polarized (LCP) and right circularly polarized (RCP) light (see Supplementary Fig. 1 for more details on these simulations). The pattern is clearly characteristic of Mie scattering.

As the CdTe nanohelices display multiple scales in their organization, and include microscale and submicrometre-scale pitch lengths comparable with the wavelengths of visible and near-infrared light, they are promising candidates for observation of not only linear chiroptical activity in scattering but also hyper (higher-order) Mie scattering rather than hyper Rayleigh scattering ${ }^{39-41}$. The TH experimental set-up to evaluate hyper Mie scattering optical activity is 

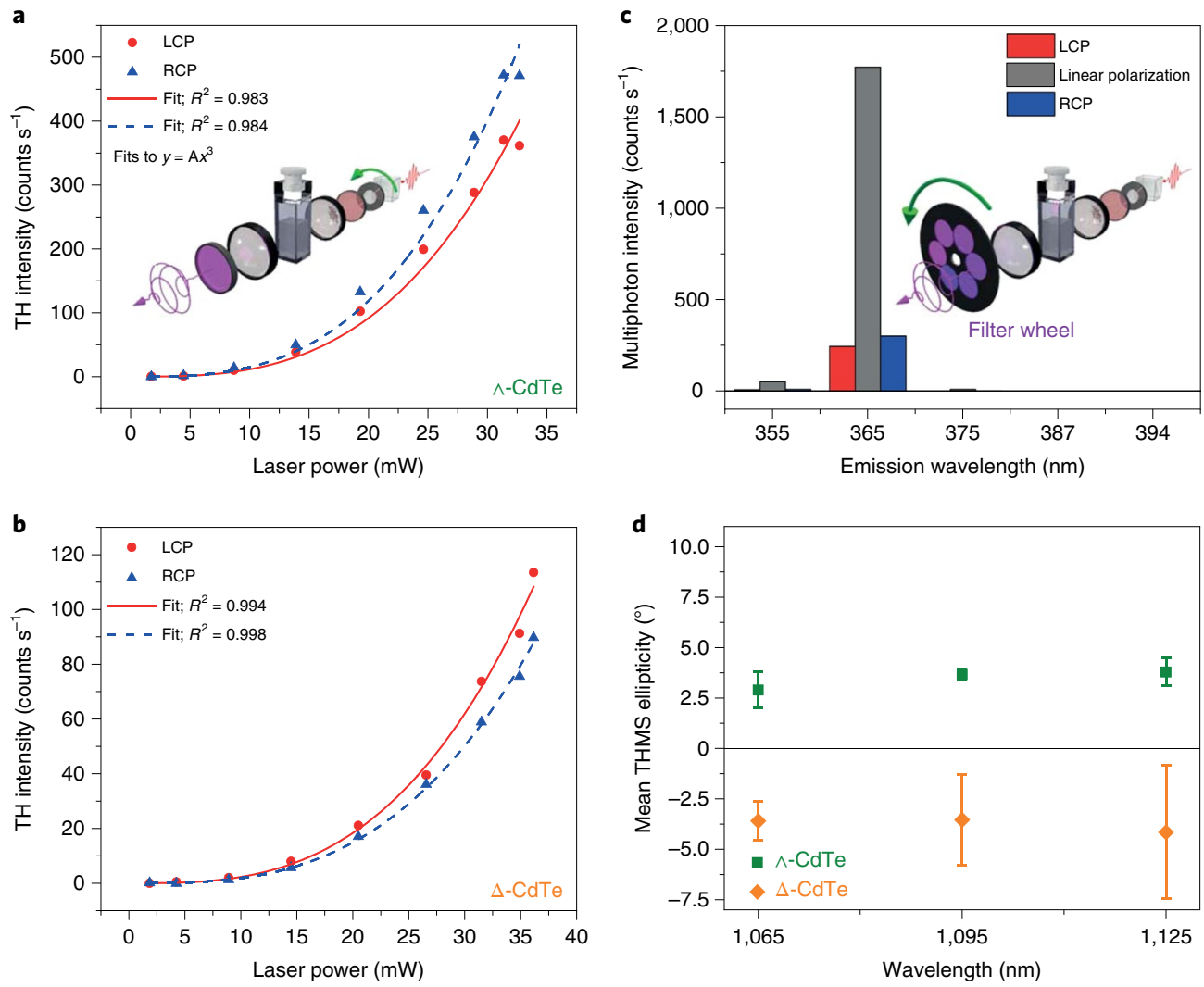

Fig. 2 | Demonstration of THMS optical activity. $\mathbf{a}, \mathbf{b}$, Third-harmonic intensity in the forward direction, as a function of incident laser power at $\lambda=1,095 \mathrm{~nm}$, is shown for LCP and RCP light from $\Lambda$-CdTe (a) and $\Delta$-CdTe (b). The symbols are experimental data points (each representing the median from 50 measurements) and the lines are fits to a cubic power-law, where $A$ is a constant. A schematic of the experiment is shown as an inset in $\mathbf{a}$. c, Multiphoton forward emission from $\Lambda$-CdTe nanohelices on illumination at 1,095 nm. The TH signal (at $365 \mathrm{~nm}$ ) clearly dominates nearby emission wavelengths for linearly polarized LCP and RCP light. d, THMS ellipticity calculated from power-dependence curves (as in $\mathbf{a}$ and $\mathbf{b}$ ) is shown for LCP and RCP light from $\Lambda$ - and $\Delta$-CdTe at three different wavelengths (see the Supplementary Information). The chiroptical response is well resolved as demonstrated by the error bars indicating standard deviation.

illustrated in Fig. 1e. The TH scattered light is measured both in the forward direction and at the right angle with respect to the direction of incident light. Briefly, femtosecond laser pulses at wavelengths of $1,065 \mathrm{~nm}, 1,095 \mathrm{~nm}$ and $1,125 \mathrm{~nm}$ are circularly polarized with an achromatic quarter-wave plate, and are focused into a cuvette made of fused quartz that contains an aqueous dispersion of CdTe nanohelices. The scattered light is then collimated and filtered to select only the TH wavelength (see Methods).

To unambiguously demonstrate a TH nature of the Mie scattered photons, it is necessary to observe, first, a cubic dependence of the measured intensity on the incident laser power and, second, the corresponding multiphoton emission spectra. Figure $2 \mathrm{a}$ shows the $\mathrm{TH}$ intensity emitted by $\Lambda$-CdTe in the forward direction (see inset) as a function of incident laser power illuminating the nanohelix dispersion at $\lambda=1,095 \mathrm{~nm}$. For both LCP and RCP light, the data fit a cubic power-law dependence with $R^{2}$ values exceeding 0.98 . There is an obvious contrast depending on the direction of circularly polarized light (CPL). Moreover, this contrast reverses for $\Delta$-CdTe nanohelices (Fig. 2b), where the data fit to a cubic power law with $R^{2}>0.99$. Similar dependences are observed for $\lambda=1,065 \mathrm{~nm}$ and $\lambda=1,125 \mathrm{~nm}$ (Supplementary Fig. 2).

Next, for light incident at $1,095 \mathrm{~nm}$, the multiphoton emission at five wavelengths is presented in Fig. 2c. Regardless of the direction of polarization, the maximum of emitted light is at $365 \mathrm{~nm}$, that is, at the TH. When the illumination wavelength changes, the position of the maximum shifts to the $\mathrm{TH}$ wavelength that corresponds to the new incident wavelength (see Supplementary Fig. 3). This impor- tant measurement rules out any contributions from other nonlinear optical effects such as four-photon luminescence or supercontinuum generation. Bearing in mind that the TH generation effect is forbidden in an isotropic liquid on illumination with $\mathrm{CPL}^{42}$, we conclude that the measured signal corresponds to TH scattering.

To quantify THMS, the ellipticity of the experimentally observed TH signal can be calculated as:

$$
\theta_{\mathrm{TH}}\left({ }^{\circ}\right)=\frac{180}{\pi} \arctan \left(\frac{\sqrt{I_{\mathrm{RCP}}^{\mathrm{TH}}}-\sqrt{I_{\mathrm{LCP}}^{\mathrm{TH}}}}{\sqrt{I_{\mathrm{RCP}}^{\mathrm{TH}}}+\sqrt{I_{\mathrm{LCP}}^{\mathrm{TH}}}}\right),
$$

where $I_{\mathrm{RCP}}^{\mathrm{TH}}$ and $I_{\mathrm{LCP}}^{\mathrm{TH}}$ are the detected $\mathrm{TH}$ intensities for incident RCP and LCP light, respectively. Figure $2 \mathrm{~d}$ shows the resultant TH ellipticities as a function of incident wavelength, for both chiral forms. The TH ellipticity seems to be relatively constant over the spectral region of $60 \mathrm{~nm}$. Crucially, the ellipticities of $\Lambda$-CdTe and $\Delta$-CdTe are clearly separated and have an opposite sign for all three wavelengths (this is not the case when achiral CdTe nanoribbons are measured, see Supplementary Fig. 4); however, this result cannot yet be attributed to THMS as it could also be due to hyper Rayleigh scattering, for example, from small regions along the nanohelices.

To better differentiate hyper Rayleigh and hyper Mie scattering effects ${ }^{43}$, TH intensity was measured at the right angle with respect to the direction of propagation of incident light (Fig. 3a). Again, an excellent cubic dependence on the incident laser power 

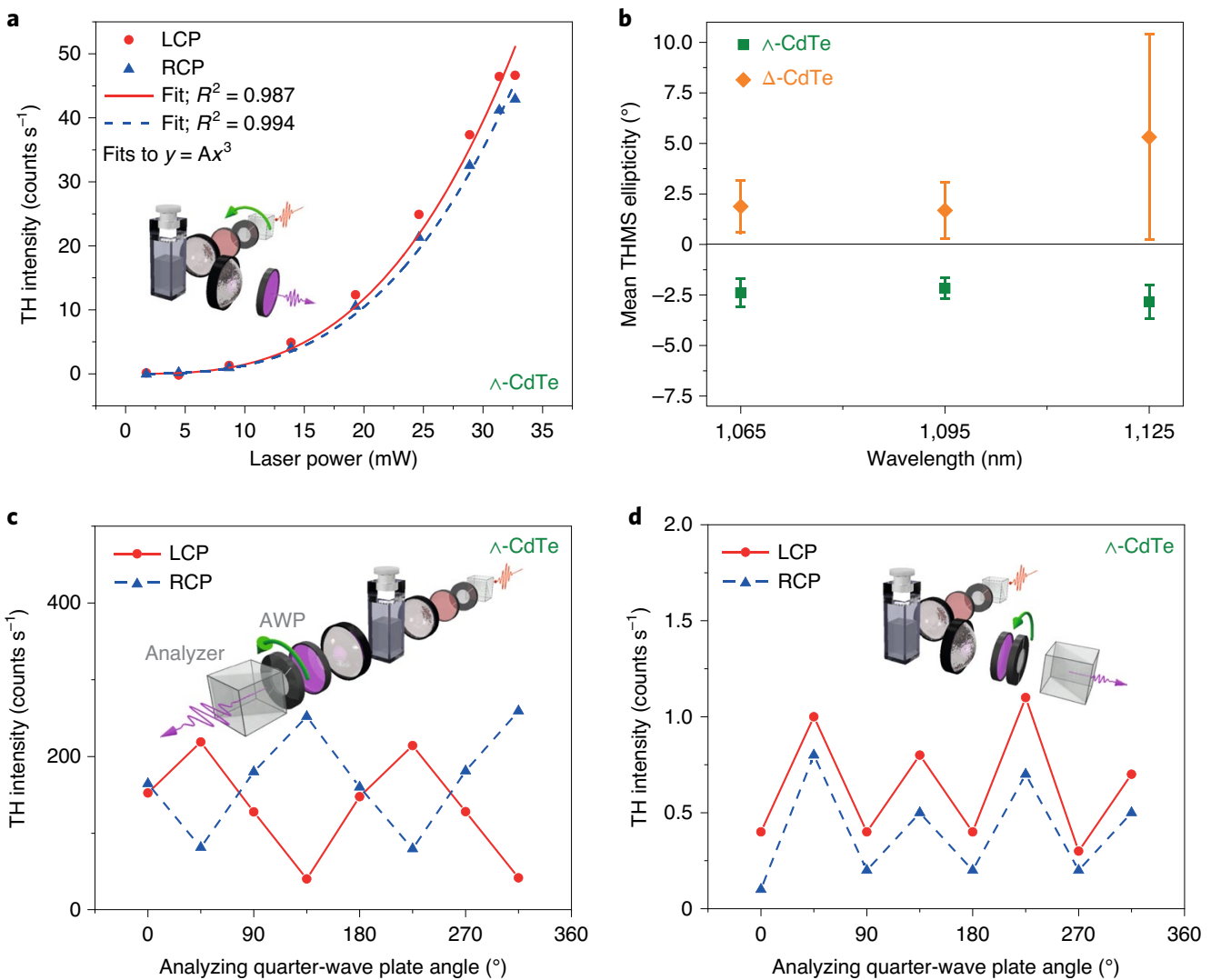

Fig. 3 Confirmation of THMS optical activity. $\mathbf{a}$, Third-harmonic intensity in the right-angle direction, as a function of incident laser power at $\lambda=1,095 \mathrm{~nm}$, is shown for LCP and RCP light from left-handed CdTe nanohelices ( $\Lambda$-CdTe). b. THMS ellipticity, calculated from power-dependence curves, is shown for LCP and RCP light from $\Lambda$ - and $\Delta$-CdTe at three different wavelengths. The chiroptical response is well resolved as demonstrated by the error bars (indicating the standard deviation). This chiroptical response is opposite to that in Fig. 2d. c, The TH intensity in the forward direction, as a function of an analysing quarter-wave plate (AWP) rotation angle for $\Lambda$-CdTe. LCP and RCP incident light produces LCP and RCP TH light, respectively. $\mathbf{d}$, The TH intensity in the $90^{\circ}$ direction, as a function of an AWP rotation angle for $\Lambda$-CdTe and $\Delta$-CdTe. LCP and RCP incident light produce linearly polarized TH light. All measurements were done for incident $1,095 \mathrm{~nm}$ light. Schematic diagrams of the experiments are shown in insets.

is observed $\left(R^{2}>0.98\right)$ and the contrast between LCP and RCP illumination reverses depending on the chirality of the CdTe nanohelices (Supplementary Fig. 5a). The multiphoton spectral signatures in Supplementary Fig. 3b and Supplementary Fig. 5b prove that the measured signal is indeed $\mathrm{TH}$ scattering and therefore a TH ellipticity spectrum can be presented in Fig. 3b. Similar trends are also observed for $\lambda=1,065 \mathrm{~nm}$ and $\lambda=1,125 \mathrm{~nm}$ illumination (Supplementary Fig. 6).

Several important differences between Figs. 2 and 3 should be noted. The intensity of $\mathrm{TH}$ light collected in the forward direction is up to ten times larger than the intensity of $\mathrm{TH}$ light collected at a right angle. This difference is consistent with Mie scattering, whereby light is emitted preferentially in the forward direction. Moreover, the chiroptical contrast in the forward direction has an opposite sign to that at right angle. Such a reversal in polarization is consistent with THMS $^{43}$. By contrast, it is not expected in the case of TH Rayleigh scattering ${ }^{44}$. Fundamentally, whereas hyper Rayleigh scattering is the result of an incoherent superposition of point-like sources, hyper Mie scattering is the result of coherent emission (from different locations on the same particle).

We next examined the polarization of the $\mathrm{TH}$ light in the forward direction and at the right angle. We modified the set-up so that the TH light passes through a rotating analysing quarter-wave plate (AWP) and then through an analyser, oriented with its transmission axis perpendicular to that of the polarizer (Fig. 3c). If the TH light is unpolarized, the measured intensity would not depend on the angle of the AWP; this is not the case. If it is linearly polarized (either along $\mathrm{S} /$ vertical or $\mathrm{P} /$ horizontal direction), the measured intensity would exhibit extrema at positions where the fast axis is along $0^{\circ}$, $90^{\circ}, 180^{\circ}$ or $270^{\circ}$; this is not the case either. If the $\mathrm{TH}$ polarization is circular, light passing through the AWP along $45^{\circ}$ or $135^{\circ}$ would have a dramatic effect, in each case the light would come out of the AWP linearly polarized; however, it would be $S$ in one case and $P$ in the other. Subsequently passing through the analyser-which is fixed along $\mathrm{P}$-would therefore result in extrema of intensity for AWP positions of $45^{\circ}, 135^{\circ}, 225^{\circ}$ and $315^{\circ}$, which is exactly what we observe in Fig. 3c. We can therefore conclude that the TH polarization in forward emission is predominantly circular and that it matches the incident polarization, regardless of the chirality of the samples. The chirality is expressed in the difference of the TH intensity: for $\Lambda$-CdTe, at AWP along $135^{\circ}$, the difference in TH intensity between RCP and LCP is larger than the difference in TH intensity between LCP and RCP at AWP along $45^{\circ}$. This behaviour is consistent with the larger frequency conversion efficiency for RCP in $\Lambda$-CdTe that is observed in Fig. 2a.

By contrast, for $\Delta-\mathrm{CdTe}$, at AWP along $135^{\circ}$, the difference in TH intensity between RCP and LCP is smaller than the difference in TH intensity between LCP and RCP at AWP along $45^{\circ}$ (see Supplementary Fig. 7a). This behaviour is consistent with the larger frequency conversion efficiency for LCP in $\Delta$-CdTe (Fig. 2b).

The same analysis can be applied to the TH emission at a right angle (Fig. $3 \mathrm{~d}$ ). Here the data present clear TH intensity minima at $0^{\circ}$, 

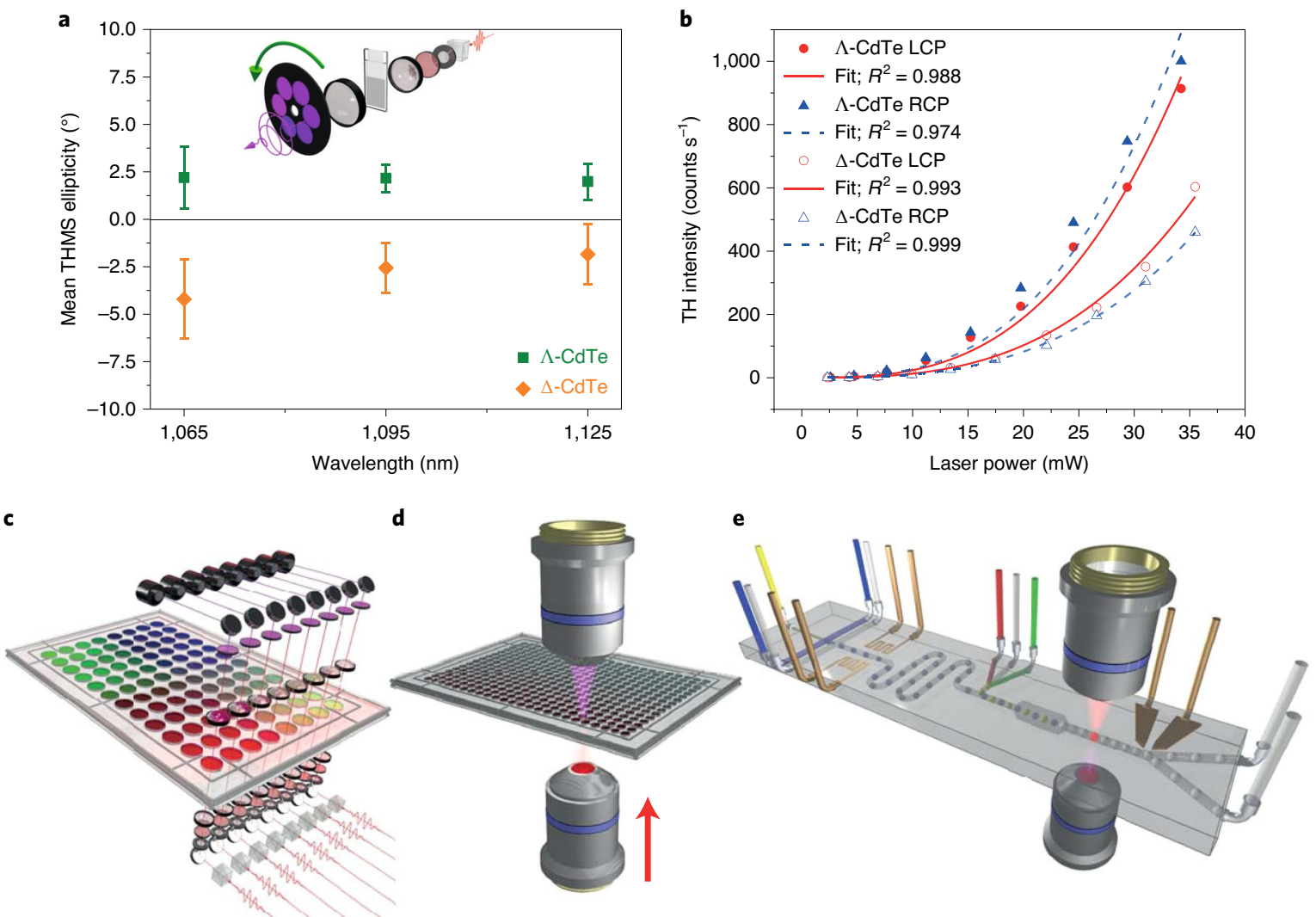

Fig. 4 | Applications of THMS for characterizing chiral nanostructures in ultrasmall volumes. a, Proof-of-principle data showing that our method can clearly differentiate between $\Lambda$-CdTe and $\Delta$-CdTe in 1-mm-thick optical cuvettes (as opposed to standard $1 \mathrm{~cm}$ cells). The THMS ellipticity was calculated from power-dependence curves measured at three different wavelengths. $\mathbf{b}$, In the same $1 \mathrm{~mm}$ cuvette, the TH intensity follows a clear cubic dependence as a function of laser power at $\lambda=1,095 \mathrm{~nm}$. Despite the small dimensions of the cuvette, the chiroptical effect for LCP and RCP, respectively, clearly reverses sign with the handedness of the samples, illustrating the potential for applications in tiny volumes. c, A schematic diagram illustrating an application of our technique as analysis tool in parallel combinatorial chemical synthesis. $\mathbf{d}$, Scanning the laser beam in an optical objective could allow applications for high-throughput experimentation, to scope reaction conditions, which generate nanomolar quantities of the desired compound in minuscule volumes (for example, $<1 \mu \mathrm{l}$ ) arrayed across a microplate. The red arrow indicates the direction of incident light. e, A schematic illustration for in-line reaction monitoring within microreactors.

$90^{\circ}, 180^{\circ}$ or $270^{\circ}$, which is consistent with predominantly S-polarized $\mathrm{TH}$ emission. Moreover, in $\Lambda$-CdTe the frequency conversion efficiency is larger for LCP, whereas in $\Delta$-CdTe (Supplementary Fig. 7b) it is larger for RCP, in agreement with the results in Fig. 3a,b.

\section{Discussion}

Third-harmonic scattering finds increasing applications as a fast and sensitive method for screening the second hyperpolarizability ${ }^{45-47}$. Due to their strong forward directionality, our results are clearly indicative of a Mie-rather than Rayleigh scattering-process. A computational model confirmed that radiation patterns from the CdTe nanohelicies with the same geometrical scale show the typical Mie scattering behaviour for electromagnetic waves with all three excitation wavelengths (Supplementary Fig. 1c-f). Accurately accounting for different scattering contributions also allows the calculated and experimental circular dichroism spectra to be matched (Fig. 1c). A multipolar decomposition of the linear optical spectra (Supplementary Fig. 8) shows that, for circularly polarized excitation, electric and magnetic dipole and quadrupole resonances can be present at both the fundamental and at the TH wavelengths. The spectral position of these resonances depends on the direction of incident light with respect to each helix and, as the particles are rotating freely, all are accessible. As demonstrated in Fig. 3c, circularly polarized fundamental light produces circularly polarized $\mathrm{TH}$ signal. It is therefore reasonable for the nonlinear conversion process to be considered as an energy coupling channel between the resonances identified in Supplementary Fig. 8, at both the fundamental and the TH.

Being cautious with assigning the physical nature of the observed optical phenomena to THMS, we want to rule out other competing mechanisms. One possibility is that our results are due to third-harmonic generation (THG) rather than Mie scattering. Both effects are coherent and would produce strong forward emission. Moreover, THG followed by a linear optical scattering process by the nanohelices would direct a small amount of light at the right angle ${ }^{48}$, which could explain the large difference in intensity between Figs. 2 and 3; however, THG is forbidden in an isotropic medium with circularly polarized light ${ }^{49}$. One reason is that on focusing a Gaussian beam in an isotropic medium, THG on one side of the focal point cancels THG on the opposite side by destructive interference, as a result of the Gouy phase shift ${ }^{50}$. Another reason is that, in the case of CPL, the axial spin angular momentum of each photon is $\pm \hbar$ (depending on the direction of CPL). For THG, as there are three incident photons the angular momentum adds up to $\pm 3 \hbar$; however, the single outgoing photon can only have a value between $\pm \hbar$; thus, in an isotropic medium such as our liquid environment, conservation of angular momentum imposes that THG for CPL illumination is forbidden in the forward direction ${ }^{49}$.

Furthermore, we can rule out any THG from the solvent or by the glass interfaces, as demonstrated in Supplementary Figs. 9 
and 10, respectively. Moreover, THG followed by linear optical Mie scattering would have resulted in the same sign of the measured ellipticities (in Figs. $2 \mathrm{~d}$ and $3 \mathrm{~b}$ ), which is clearly not the case. Furthermore, THG followed by linear chiroptical effects such as circular dichroism could have also exhibited optical rotation. In Supplementary Section 11 we illuminated the sample with linearly polarized light and tested for any such optical rotation; none was found (see Supplementary Fig. 11). We can therefore rule out THG and, furthermore, Fig. $2 \mathrm{c}$ and Supplementary Fig. 3a demonstrate the absence of any competing multiphoton luminescence or supercontinuum effects.

Mie scattering takes place at a scale that is between point scatterers (Rayleigh scattering) and geometric optics. At the microscopic scale, the induced electric dipole moment can be written as:

$$
\mu_{i}=\alpha_{i j} E_{j}+\beta_{i j k} E_{j} E_{k}+\gamma_{i j k l} E_{j} E_{k} E_{l}+\cdots
$$

where $i, j, k$ and $l$ can represent any of the Cartesian directions in the $x, y, z$ coordinate system of the helix, $\alpha$ is the polarizability tensor, $\beta$ is the first hyperpolarizability tensor, $\gamma$ is the second hyperpolarizability tensor and $E_{j}$ is the electric field component at the fundamental frequency of light along the $j$ Cartesian direction of the helix coordinate system (that can take $x, y$ or $z$ values). The induced dipole moment per unit volume at the TH can then be written as:

$$
P_{I}(3 \omega)=\chi_{I J K L} E_{J}(\omega) E_{K}(\omega) E_{L}(\omega),
$$

where $\chi_{I J K L}$ is the macroscopic third-order susceptibility and $I$, $J, K$ and $L$ represent any of the Cartesian directions in the $X$, $Y, Z$ coordinate system of the laboratory (Fig. 1e). The expressions at the microscopic and macroscopic scale are related by $\chi_{I J K L}=\frac{N \gamma_{i j k l}}{\varepsilon_{0}}\left\langle a_{i I} a_{j J} a_{k K} a_{l L}\right\rangle$, where $a_{i I}, a_{j J}, a_{k K}$ and $a_{l L}$ are the direction cosines linking the two frames of reference and $\langle\ldots\rangle$ denotes a statistical average of the orientation of the scatterers, whose number is $N$; $\varepsilon_{0}$ is the permittivity of vacuum.

For an isotropic chiral medium, such as an enantiopure suspension of nanohelices, $\chi_{I J K L}$ is not zero. The isotropic chiral symmetry group belongs to one of the seven Curie limiting point groups of anisotropic materials ${ }^{51}$. It is denoted by $\infty \infty$ in Hermann-Mauguin, $\infty / \infty$ in Shubnikov, $\mathbf{K}$ in Schoenflies and $\infty / \infty 2$ in international notations. It has three independent tensor components, $\chi_{I I J J}, \chi_{I J I}, \chi_{I I I J}$, and they are related by $\chi_{I I I I}=\chi_{I I J}+\chi_{I J I I}+\chi_{I I I}$, where the indices I,J can take any of the laboratory frame coordinates $X, Y$ or $Z$ and where $I \neq J$ (ref. ${ }^{52}$ ).

The intensity at the $\mathrm{TH}$ is then:

$$
I(3 \omega) \propto N\left\langle\left|\chi_{\mathrm{THS}}\right|^{2}\right\rangle I^{3}(\omega) .
$$

Generally, in the forward $X$ detection, the third harmonic scattering (THS) intensity is $\left\langle\left|\chi_{\mathrm{THS}}\right|^{2}\right\rangle=\left\langle\left|\chi_{Z J K L}\right|^{2}\right\rangle+\left\langle\left|\chi_{Y J K L}\right|^{2}\right\rangle$, where, for CPL incident light, $J, K$ and $L$ can be either $Y$ or $Z$ but not $X$. For an isotropic chiral medium, there are non-zero tensor components in both $\left\langle\left|\chi_{Z J K L}\right|^{2}\right\rangle$ and $\left\langle\left|\chi_{Y J K L}\right|^{2}\right\rangle$; TH polarization is thus allowed along both the $Z$ and $Y$ directions, which leads to the TH intensities we observe. In Fig. 3c, the CPL TH light has both $Z$ and $Y$ components.

By contrast, in the $Y$ direction (at a right angle with respect to the incident beam), $\left\langle\left|\chi_{T H S}\right|^{2}\right\rangle=\left\langle\left|\chi_{X J K L}\right|^{2}\right\rangle+\left\langle\left|\chi_{Z J K L}\right|^{2}\right\rangle$, where, for CPL illumination, $J, K$ and $L$ can again take $Y$ or $Z$ values but not $X$. As a consequence, in an isotropic chiral medium, only the $\left\langle\left|\chi_{Z J K L}\right|^{2}\right\rangle$ terms can contribute, which means that the TH can only be vertically polarized, as we see in Fig. $3 \mathrm{~d}$.

We next consider the microscopic hyperpolarizabilities. The symmetry group of the nanohelices is $D_{2}$ in Schoenflies and 222 in
Hermann-Maugin notations. It has 21 independent tensor components, 3 with all indices equal and 18 with equal indices in pairs ${ }^{53}$.

We need to express $\mu_{i}$ in the laboratory coordinate system $X$, $Y, Z$. We define the angles $\varphi$ and $\phi$, such as $Z$ is along $\varphi=0$ and $\mu_{z}=\cos (\varphi), \mu_{Y}=\sin (\varphi) \cos (\phi), \mu_{X}=\sin (\varphi) \sin (\phi)$. The statistical average for individual $\chi_{I J K L}$ components is then calculated ${ }^{54}$ by:

$$
\left\langle\chi_{I J K L}\right\rangle=\frac{1}{4 \pi} \int_{0}^{2 \pi} \int_{0}^{\pi} \sum_{i, j, k, l}\left(a_{I i} a_{J j} a_{K k} a_{L l}\right) \gamma_{i j k l} \sin (\varphi) d \varphi d \phi
$$

For the $D_{2}$ symmetry group, we use $\frac{1}{4 \pi} \int_{0}^{2 \pi} \int_{0}^{\pi}(\cos (\varphi))^{4} \sin (\varphi) d \varphi d \phi=\frac{1}{5}$ to calculate the

$\gamma_{i j i i} \quad$ terms $\quad$ and $\frac{1}{4 \pi} \int_{0}^{2 \pi} \int_{0}^{\pi}(\cos (\varphi))^{2}(\sin (\varphi))^{2}(\cos (\phi))^{2} \sin (\varphi) d \varphi d \phi=\frac{1}{15}$ to calculate the

$\gamma_{i i j j}, \gamma_{i j i j}$ and $\gamma_{i j i j}$ terms.

We can therefore write:

$$
\left\langle\chi_{Z Z Z Z}\right\rangle=\frac{1}{5} \sum_{i} \gamma_{i i i i}+\frac{1}{15} \sum_{i, j}\left(\gamma_{i i j j}+\gamma_{i j i j}+\gamma_{i j j i}\right)
$$

Similar values can be calculated for the other macroscopic susceptibilities; however, as there are 21 microscopic hyperpolarizability tensor elements, each of them being complex, the number of parameters is too large for intuitive understanding.

For simplicity, we adopt an alternative notation, with effective chiral and achiral parts of the macroscopic susceptibility. The latter includes any contributions from the solvent or from degraded, achiral CdTe particles. In the following expression, upon reversing the sample chirality the chiral part changes sign but the achiral part does not (changing the direction of CPL has an equivalent effect):

$$
\left|\chi_{I J K L}\right|_{\mathrm{LCP} / \mathrm{RCP}}^{2}=\left|\chi_{I J K L}^{\text {achiral }} \pm \chi_{I J K L}^{\text {chiral }}\right|^{2}
$$

In this case, the $\mathrm{TH}$ ellipticity is proportional to:

$$
\frac{\left|\chi_{I J K L}\right|_{\mathrm{RCP}}^{2}-\left|\chi_{I J K L}\right|_{\mathrm{LCP}}^{2}}{\left|\chi_{I J K L}\right|_{\mathrm{RCP}}^{2}+\left|\chi_{I J K L}\right|_{\mathrm{LCP}}^{2}}=\frac{-\left|\chi_{I J K L}^{\text {chiral }}\right|\left|\chi_{I J K L}^{\text {achiral }}\right|}{\left|\chi_{I J K L}^{\text {achiral }}\right|^{2}+\left|\chi_{\text {IJKL }}^{\text {chiral }}\right|^{2}} \cos (\sigma),
$$

where $\sigma$ is the phase angle between the complex chiral and achiral effective susceptibilities. When $\left|\chi_{I J K L}^{\text {chiral }}\right| \ll\left|\chi_{I J K L}^{\text {achiral }}\right|$, this quantity is directly proportional to the chiral part of the nonlinear susceptibility. When $\left|\chi_{I J K L}^{\text {chiral }}\right| \gg\left|\chi_{I J K L}^{\text {achiral }}\right|$, the measured quantity is inversely proportional to the chiral part of the nonlinear susceptibility. When $\left|\chi_{I J K L}^{\text {chiral }}\right| \approx\left|\chi_{I J K L}^{\text {achiral }}\right|$, the quantity is constant and proportional to $\cos (\sigma)$. When $\sigma=\pi / 2$, the chiral and achiral susceptibility parts are out of phase and no chiroptical effect can be measured. The implication also is that chiroptical effects can only be measured when the chiral and achiral tensor components interfere. As the sign of the $\mathrm{TH}$ ellipticity depends on the phase angle between numerator and denominator, we can conclude that $\sigma$ is opposite for the emissions along $X$ (Fig. 2d) and $Y$ (Fig. 3b).

To demonstrate the applicability of our technique in volumes of practical relevance for 1,536-well microplates, we performed a THMS characterization of $\Lambda$-CdTe and $\Delta$-CdTe in cuvettes with a $1 \mathrm{~mm}$ optical path length, which is around ten-times thinner than standard 1-cm-thick cuvettes (the four interfaces of these cuvettes are still well resolved by our set-up, see Supplementary Fig. 12). For a conservative estimate, the volume of illumination can then be approximated to $1 \mathrm{~mm}^{3}=10^{-9} \mathrm{~m}^{3}=1 \mu$ l. Figure 4 a shows the THMS ellipticity spectra obtained from power-dependence measurements, such as those in Fig. 4b (the power-dependence curves measured at $1,065 \mathrm{~nm}$ and $1,125 \mathrm{~nm}$ can be found in Supplementary Fig. 13). 
These proof-of-principle data show that our technique can clearly distinguish the two chiral forms when the path length is reduced by one order of magnitude (compared with Fig. 2), which asserts the inherent scalability of our method. We believe that with further development and by using a calibration procedure, the technique could also estimate enantiopurity. Parallel processing (Fig. 4c) would allow the screening of combinatorial chemistry microplates $^{55}$. In miniature arrays, it is possible to use optical objectives and scan the beam across the array (Fig. 4d). These arrays would allow the study of various chemical reactions that lead to a change of the circular dichroism spectrum of the sample. For instance, using the nanohelices presented here, the ionic replacement of $\mathrm{Cd}^{2+}$ with $\mathrm{Pb}^{2+}$ would be very interesting to perform. On dispersing the CdTe nanohelices in a solution of $\mathrm{Pb}\left(\mathrm{NO}_{3}\right)_{2}$, lead progressively substitutes the cadmium atoms. This process dramatically affects the circular dichroism in the linear optical regime and, using a microplate, it could be monitored across various wells with THMS optical activity, as shown in Fig. 4d. Following further development and optimization (in terms of illumination power, pulse duration, repetition rate, wavelength, volume in focus, geometry and material composition of the scatterers, detection electronics, signal noise reduction methods and so on), our technique could be sped up and could also find applications as an in-line analysis tool for continuous flow microreactors $^{56}$ (see Fig. 4e). Finally, it should be emphasized that a more accurate estimate of the volume of illumination (on integrating the beam area around the beam waist $\left(\omega_{0}=6.15 \times 10^{-6} \mathrm{~m}\right)$ from - to + the Rayleigh range $\left.\left(z_{\mathrm{R}}=1.44 \times 10^{-4} \mathrm{~m}\right)\right)$ is around $4.56 \times 10^{-14} \mathrm{~m}^{3}$, which is five orders of magnitude smaller than the $1 \mu \mathrm{l}$ in 1,536 -well microplates. Moreover, using an optical objective instead of a lens can further reduce the illumination focal volume by several orders of magnitude. For a long working distance objective of $50 \times$, the focal volume was estimated to be around $3 \times 10^{-17} \mathrm{~m}^{3}$ (in the shape of a rotational ellipsoid with its axes $2.3 \mu \mathrm{m}$ and $11 \mu \mathrm{m}$ long), by recording a multiphoton microscope Z-stack scan of a fluorescent nanosphere of around $200 \mathrm{~nm}$ in diameter, with an illumination wavelength of $730 \mathrm{~nm}$; this volume is eight orders of magnitude less than $1 \mu \mathrm{l}$ (ref. ${ }^{41}$ ). Considering the shape of the illumination volume the pathlength could potentially be reduced well below $100 \mu \mathrm{m}$ with suitable illumination optics.

This study demonstrates the fundamental possibility of nonlinear chiroptical activity for Mie scattering, represented by THMS. Carefully ruling out contributions from THG, multiphoton luminescence and supercontinuum generation, this effect was observed for semiconductor $\mathrm{CdTe}$ nanohelices at three different wavelengths. The magnitude of THMS is large, displaying a TH ellipticity approximately $3^{\circ}$ from an illumination volume as small as $V_{\text {focus }} \approx 4.56 \times 10^{-14} \mathrm{~m}^{3}$. In agreement with physics of Mie scattering, the effect is highly directional, with approximately ten times more TH light detected in the forward direction than in the sideways direction.Although the $\mathrm{TH}$ emission is circularly polarized in the forward direction, it is linearly polarized (vertically) in the right-angle direction. In both cases, a clear chiroptical response is observed, with a sign reversal, which indicates that emission in the forward direction and at the right angle is due to different sets of $\mathrm{TH}$ effective susceptibility tensor components, whereby the phase between the chiral and achiral parts changes sign depending on the direction of emission. Our results open the way for chiroptical characterization of semiconductor and other chiral non-metallic particles in volumes potentially of the order of $10^{-17} \mathrm{~m}^{3}$ (ref. ${ }^{41}$ ). Such miniaturized reaction volume offers a technological link between chemical synthesis and data science requirements exploration of chiral nanostructures. Furthermore, it can be potentially extended to chiral nanostructured particles with multiscale organization and similar dimensions, such as extracellular vesicles, that are widely investigated nowadays for cancer detection. Together with inorganic nanoparticle labelling, high THMS intensity offers a pathway to detect and digitize the presence of rare extracellular vesicles with microfluidics ${ }^{57}$.

\section{Online content}

Any methods, additional references, Nature Research reporting summaries, source data, extended data, supplementary information, acknowledgements, peer review information; details of author contributions and competing interests; and statements of data and code availability are available at https://doi.org/10.1038/ s41566-021-00916-6.

Received: 4 August 2021; Accepted: 20 October 2021;

Published online: 13 January 2022

\section{References}

1. Himanen, L., Geurts, A., Foster, A. S. \& Rinke, P. Data-driven materials science: status, challenges, and perspectives. Adv. Sci. 6, 1900808 (2019).

2. Curtarolo, S. et al. The high-throughput highway to computational materials design. Nat. Mater. 12, 191-201 (2013)

3. Coley, C. W., Eyke, N. S. \& Jensen, K. F. Autonomous discovery in the chemical sciences part I: progress. Angew. Chemie Int. Ed. 59, 22858-22893 (2020)

4. Eyke, N. S., Koscher, B. A. \& Jensen, K. F. Toward machine learning-enhanced high-throughput experimentation. Trends Chem. 3, 120-132 (2021).

5. Cernak, T. Synthesis in the chemical space age. Chem 1, 6-9 (2016).

6. Kutchukian, P. S. et al. Chemistry informer libraries: a chemoinformatics enabled approach to evaluate and advance synthetic methods. Chem. Sci. 7, 2604-2613 (2016).

7. Shen, Y. et al. Automation and computer-assisted planning for chemical synthesis. Nat. Rev. Methods Prim. 1, 23 (2021).

8. Jiang, W. et al. Emergence of complexity in hierarchically organized chiral particles. Science 368, 642-648 (2020).

9. Lee, H.-E. et al. Amino-acid- and peptide-directed synthesis of chiral plasmonic gold nanoparticles. Nature 556, 360-365 (2018).

10. Ben-Moshe, A. et al. Enantioselective control of lattice and shape chirality in inorganic nanostructures using chiral biomolecules. Nat. Commun. 5, 4302 (2014).

11. Valev, V. K., Baumberg, J. J., Sibilia, C. \& Verbiest, T. Chirality and chiroptical effects in plasmonic nanostructures: fundamentals, recent progress, and outlook. Adv. Mater. 25, 2517-2534 (2013)

12. Kotov, N. A. Inorganic nanoparticles as protein mimics. Science 330, 188-189 (2010).

13. Srivastava, S. et al. Light-controlled self-assembly of semiconductor nanoparticles into twisted ribbons. Science 327, 1355-1359 (2010).

14. Buitrago Santanilla, A. et al. Nanomole-scale high-throughput chemistry for the synthesis of complex molecules. Science 347, 49-53 (2015).

15. Belkin, M. A., Han, S. H., Wei, X. \& Shen, Y. R. Sum-frequency generation in chiral liquids near electronic resonance. Phys. Rev. Lett. 87, 113001 (2001).

16. Donskoi, S. M. \& Makarov, V. A. Five-wave mixing $\omega b=\omega 1+\omega 1+\omega 1-\omega 2$ in the bulk of a chiral liquid. J. Raman Spectrosc. 31, 779-784 (2000).

17. Romero, L. C. D., Meech, S. R. \& Andrews, D. L. Five-wave mixing in molecular fluids. J. Phys. B At. Mol. Opt. Phys. 30, 5609-5619 (1997).

18. De Boni, L., Toro, C. \& Hernández, F. E. Synchronized double L-scan technique for the simultaneous measurement of polarization-dependent two-photon absorption in chiral molecules. Opt. Lett. 33, 2958 (2008).

19. Mesnil, H. \& Hache, F. Experimental evidence of third-order nonlinear dichroism in a liquid of chiral molecules. Phys. Rev. Lett. 85, 4257-4260 (2000).

20. Markowicz, P. P. et al. Modified Z-scan techniques for investigations of nonlinear chiroptical effects. Opt. Exp. 12, 5209 (2004).

21. Jain, P. K., Lee, K. S., El-Sayed, I. H. \& El-Sayed, M. A. Calculated absorption and scattering properties of gold nanoparticles of different size, shape, and composition: applications in biological imaging and biomedicine. J. Phys. Chem. B 110, 7238-7248 (2006).

22. Jain, P. K., Huang, X., El-Sayed, I. H. \& El-Sayed, M. A. Noble metals on the nanoscale: optical and photothermal properties and some applications in imaging, sensing, biology, and medicine. Acc. Chem. Res. 41, 1578-1586 (2008).

23. Wang, Z., Cheng, F., Winsor, T. \& Liu, Y. Optical chiral metamaterials: a review of the fundamentals, fabrication methods and applications. Nanotechnology 27, 412001 (2016).

24. Ren, M., Plum, E., Xu, J. \& Zheludev, N. I. Giant nonlinear optical activity in a plasmonic metamaterial. Nat. Commun. 3, 833 (2012).

25. Tang, Y. et al. Nano-Kirigami metasurface with giant nonlinear optical circular dichroism. Laser Photon. Rev. 14, 2000085 (2020). 
26. Ma, W. et al. Chiral inorganic nanostructures. Chem. Rev. 117, 8041-8093 (2017).

27. Guerrero-Martínez, A., Alonso-Gómez, J. L., Auguié, B., Cid, M. M. \& Liz-Marzán, L. M. From individual to collective chirality in metal nanoparticles. Nano Today 6, 381-400 (2011)

28. Gramotnev, D. K. \& Bozhevolnyi, S. I. Plasmonics beyond the diffraction limit. Nat. Photon. 4, 83-91 (2010).

29. Menon, V. M., Deych, L. I. \& Lisyansky, A. A. Towards polaritonic logic circuits. Nat. Photon. 4, 345-346 (2010).

30. Miller, D. A. B. Are optical transistors the logical next step? Nat. Photon. 4, 3-5 (2010).

31. Ali, R., Dutra, R. S., Pinheiro, F. A., Rosa, F. S. S. \& Maia Neto, P. A. Theory of optical tweezing of dielectric microspheres in chiral host media and its applications. Sci. Rep. 10, 16481 (2020).

32. Shcherbakov, M. R. et al. Enhanced third-harmonic generation in silicon nanoparticles driven by magnetic response. Nano Lett. 14, 6488-6492 (2014).

33. Smirnova, D. A., Khanikaev, A. B., Smirnov, L. A. \& Kivshar, Y. S. Multipolar third-harmonic generation driven by optically induced magnetic resonances. ACS Photonics 3, 1468-1476 (2016).

34. Gandolfi, M., Tognazzi, A., Rocco, D., De Angelis, C. \& Carletti, L. Near-unity third-harmonic circular dichroism driven by a quasibound state in the continuum in asymmetric silicon metasurfaces. Phys. Rev. A 104, 023524 (2021).

35. Grinblat, G., Li, Y., Nielsen, M. P., Oulton, R. F. \& Maier, S. A. Enhanced third harmonic generation in single germanium nanodisks excited at the anapole mode. Nano Lett 16, 4635-4640 (2016).

36. Smirnova, D. \& Kivshar, Y. S. Multipolar nonlinear nanophotonics. Optica 3, 1241 (2016)

37. Yan, J. et al. Self-assembly of chiral nanoparticles into semiconductor helices with tunable near-infrared optical activity. Chem. Mater. 32, 476-488 (2020).

38. Xia, Y. et al. Self-assembly of self-limiting monodisperse supraparticles from polydisperse nanoparticles. Nat. Nanotechnol. 7, 479-479 (2012).

39. Collins, J. T. et al. First observation of optical activity in hyper-Rayleigh scattering. Phys. Rev. X 9, 011024 (2019).

40. Verreault, D. et al. Hyper-Rayleigh scattering as a new chiroptical method: uncovering the nonlinear optical activity of aromatic oligoamide foldamers. $J$. Am. Chem. Soc. 142, 257-263 (2020).

41. Ohnoutek, L. et al. Single nanoparticle chiroptics in a liquid: optical activity in hyper-Rayleigh scattering from Au helicoids. Nano Lett 20, 5792-5798 (2020).

42. Bey, P. P., Giuliani, J. F. \& Rabin, H. Linear and circular polarized laser radiation in optical third harmonic generation. Phys. Lett. A 26, 128-129 (1968).
43. Dewitz, J. P., Hübner, W. \& Bennemann, K. H. Theory for nonlinear Mie-scattering from spherical metal clusters. Zeitschrift für Phys. D 37, 75-84 (1996).

44. Ohnoutek, L. et al. Optical activity in third-harmonic rayleigh scattering: a new route for measuring chirality. Laser Photon. Rev. 2100235 (2021); https:// doi.org/10.1002/lpor.202100235

45. Van Steerteghem, N., Clays, K., Verbiest, T. \& Van Cleuvenbergen, S. Third-Harmonic Scattering for Fast and Sensitive Screening of the Second Hyperpolarizability in Solution. Anal. Chem. 89, 2964-2971 (2017).

46. Moris, M. et al. Harmonic light scattering study reveals structured clusters upon the supramolecular aggregation of regioregular poly(3-alkylthiophene). Commun. Chem. 2, 130 (2019).

47. Ford, J. S. \& Andrews, D. L. Molecular tensor analysis of third-harmonic scattering in liquids. J. Phys. Chem. A 122, 563-573 (2018).

48. Shelton, D. P. Third harmonic scattering in liquids. J. Chem. Phys. 149, 224504 (2018)

49. Tang, C. L. \& Rabin, H. Selection rules for circularly polarized waves in nonlinear optics. Phys. Rev. B 3, 4025-4034 (1971).

50. Clay, G. O. et al. Spectroscopy of third-harmonic generation: evidence for resonances in model compounds and ligated hemoglobin. J. Opt. Soc. Am. B 23, 932 (2006)

51. Shubnikov, A. V. in Crystal Symmetries Vol. 16, 357-364 (Elsevier, 1988).

52. Grigoriev, K. S., Kuznetsov, N. Y., Cherepetskaya, E. B. \& Makarov, V. A. Second harmonic generation in isotropic chiral medium with nonlocality of nonlinear optical response by heterogeneously polarized pulsed beams. Opt. Express 25, 6253 (2017).

53. Boyd, R. W. Nonlinear Optics (Elsevier, 2003); https://doi.org/10.1016/ B978-0-12-121682-5.X5000-7

54. Multian, V. V. et al. Averaged third-order susceptibility of ZnO nanocrystals from third harmonic generation and third harmonic scattering. Opt. Mater. (Amst). 84, 579-585 (2018).

55. Takatsy, G. A rapid and accurate method for serial dilutions. Kiserl Orvostud 5, 393-397 (1950)

56. Galaverna, R., Ribessi, R. L., Rohwedder, J. J. R. \& Pastre, J. C. Coupling continuous flow microreactors to microNIR spectroscopy: ultracompact device for facile in-line reaction monitoring. Org. Process Res. Dev. 22, 780-788 (2018).

57. $\mathrm{Qu}, \mathrm{Z}$. et al. Metal-bridged graphene-protein supraparticles for analog and digital nitric oxide sensing. Adv. Mater. 33, 2007900 (2021).

Publisher's note Springer Nature remains neutral with regard to jurisdictional claims in published maps and institutional affiliations.

(c) The Author(s), under exclusive licence to Springer Nature Limited 2022 


\section{Methods}

Materials. All chemicals used in this study were in analytical grade or the highest purity available. Aluminium telluride $\left(\mathrm{Al}_{2} \mathrm{Te}_{3}\right)$ used as a source of hydrogen telluride gas was purchased from Materion (product no. 122704). Cadmium perchlorate hexahydrate was purchased from Alfa Aesar (product no. 12936). L- and D-cysteine hydrochloride monohydrate (product nos. C7880 and C8005, respectively), thioglycolic acid (product no. 528056), sodium hydroxide $(\mathrm{NaOH})$, hydrochloric acid $(\mathrm{HCl})$ and methanol were purchased from Sigma Aldrich. Deionized water was prepared with Milli-Q water purification system (Millipore).

Synthesis of cadmium telluride nanoparticles (CdTe NPs). The CdTe NPs were synthesized by following a method from previous publications after some modification $^{59}$. For chiral CdTe NPs, an aqueous solution of mixture of cadmium perchlorate hexahydrate $\left(\mathrm{Cd}\left(\mathrm{ClO}_{4}\right)_{2} \cdot 6 \mathrm{H}_{2} \mathrm{O}\right)$ and L- or D-cysteine (L-/D-Cys) hydrochloride monohydrate is prepared with final concentration of $18.75 \mathrm{mM}$ and $45 \mathrm{mM}$ in $125 \mathrm{ml}$ of deionized water. Then the $\mathrm{pH}$ of the mixture solution was adjusted to 11.2 by adding $1 \mathrm{M}$ sodium hydroxide $(\mathrm{NaOH})$. The mixture solution was placed in a $250 \mathrm{ml}$ three-neck round-bottomed flask and connected with another $50 \mathrm{ml}$ three-neck round-bottomed flask containing $0.1 \mathrm{~g}$ of aluminium telluride powder $\left(\mathrm{Al}_{2} \mathrm{Te}\right)$. These synthesis reaction vessels were purged with nitrogen gas for $30 \mathrm{~min}$ with vigorous stirring for good mixture of the solution. Hydrogen telluride gas was generated by injecting $0.5 \mathrm{M}$ hydrogen sulphide into the small flask containing $\mathrm{Al}_{2} \mathrm{Te}$ powder and slowly purged into the cadmium precursor and the ligand mixture solution. The solution was then refluxed under nitrogen purging for about $10 \mathrm{~h}$ to obtain the chiral CdTe NPs. The achiral CdTe NPs were prepared following the same method described above but by using thioglycolic acid (TGA) as the surface ligand source $(18.75 \mathrm{mM})$ for the cadmium precursor mixture solution. After purging the hydrogen telluride gas slowly, the solution was refluxed for $1 \mathrm{~h}$. The as-made NP dispersion were stored in a dark bottle before assembly processes with aging requirements as described in the assembly method section.

Preparation of CdTe nanohelices and achiral CdTe nanoribbons colloids. Self-assembly of nanohelices. The CdTe NPs carrying $L$ - or $D$-Cys were assembled into helical structure with a modified method from previous works ${ }^{37,60}$. The dispersions of synthesized chiral CdTe NPs were aged at room temperature in the dark for three to five days before self-assembly; $20 \mu \mathrm{l}$ of $0.1 \mathrm{M} \mathrm{Cd}\left(\mathrm{ClO}_{4}\right)_{2} \cdot 6 \mathrm{H}_{2} \mathrm{O}$ was injected into the aged $500 \mu \mathrm{l}$ of chiral NPs and the $\mathrm{pH}$ adjusted to 8 with $1 \mathrm{M}$ hydrochloric acid solution. Depending on batch, the solution was then mixed with $1 \mathrm{ml}$ or $1.5 \mathrm{ml}$ of methanol and kept for three days in room light conditions. The self-assembled colloid was centrifuged for $3 \mathrm{~min}$ at 6,000 r.p.m. and the black precipitates were redispersed into deionized water. The resulting solution was treated with the same washing process three times to ensure obtaining purified nanohelices colloids. To avoid aggregation of the nanohelices, final precipitates were redispersed in $0.05 \mathrm{wt} \%$ of sodium dodecyl sulfate (SDS) solution and sonicated for approximately $1 \mathrm{~min}$.

Self-assembly of achiral nanoribbons. The CdTe NPs capped by TGA were assembled into achiral nanoribbons by modifying the method described above for assembly of nanohelicies. Without adding $\mathrm{Cd}\left(\mathrm{ClO}_{4}\right)_{2} \cdot 6 \mathrm{H}_{2} \mathrm{O}$, the $\mathrm{pH}$ of the aged $500 \mu \mathrm{l}$ of TGA-CdTe NPs was directly adjusted to 8 with $1 \mathrm{M}$ hydrochloric acid solution, mixed with $1.5 \mathrm{ml}$ of methanol and kept for three days in room light conditions. The nanoribbon solution treated with the same washing process of nanohelicies was redispersed in $0.05 \mathrm{wt} \%$ of SDS solution and sonicated for approximately $1 \mathrm{~min}$.

Linear optical characterization. Suspensions of CdTe nanohelices in fused quartz cuvettes were characterized in an Applied Photophysics Chirascan circular dichroism spectrometer. Two photomultiplier tubes (PMTs) were used as detectors-each of them designed for measurements in a different spectral region. Measured circular dichroism spectrum of the reference sample (a cuvette with $0.2 \%$ SDS) was subtracted from the spectra of the investigated samples. The path length in the cuvette was $10 \mathrm{~mm}$. The time-per-point was set to $0.5 \mathrm{~s}$ and the step to $1 \mathrm{~nm}$. Each spectrum was measured three times before taking an average. The bandwidth was set to $2 \mathrm{~nm}$ in the $300-600 \mathrm{~nm}$ spectral region and to $40 \mathrm{~nm}$ in the $600-1,130 \mathrm{~nm}$ region.

Nonlinear experiments. An optical parametric oscillator pumped by a Ti:sapphire laser was used as the laser source for the nonlinear experiments. The laser pulses were produced by the optical parametric oscillator with a repetition rate of $80 \mathrm{MHz}$ and had a width of $200 \mathrm{fs}$. An optical chopper with $50 \%$ duty cycle modulated the laser beam at a frequency of $246 \mathrm{~Hz}$. An achromatic half-wave plate designed to work in the $690-1,200 \mathrm{~nm}$ spectral range was placed before an uncoated Glan-Laser calcite polarizer for power control. After passing through the polarizer (transmission axis oriented vertically), an achromatic quarter-wave plate (design wavelength range $690-1,200 \mathrm{~nm}$ ) controlled the polarization state of the laser beam. Left circularly polarized light is defined from the point of view of the source, looking along the direction of propagation, such that the electric field of light traces a helix in space that rotates following the positive trigonometric direction. A pair of longpass filters removed light with wavelengths below the filters' cut-on wavelength $(1,000 \mathrm{~nm})$ from the beam. A coated achromatic doublet lens (design wavelength range $1,050-1,700 \mathrm{~nm}$, focal length $30 \mathrm{~mm}$ ) focused the beam into a fused quartz cuvette containing the samples.

In experiments measuring scattering at a right angle, an anti-reflection coated 25.4-mm lens was used to collect scattered light. Another anti-reflection coated lens (focal length $200 \mathrm{~nm}$ ) focused the collected light onto the photocathode of a PMT. In experiments performed in transmission geometry, an anti-reflection coated 25.4-mm focal-length lens was placed after the cuvette and was followed by a coloured glass bandpass filter (transmission in the $335-610 \mathrm{~nm}$ region), 3 UV-enhanced $\mathrm{Al}$ mirrors, and a 200 - $\mathrm{mm}$ focal-length lens, which focused light onto the photocathode of a PMT.

To investigate the polarization of the THMS light, a superachromatic quarter-wave plate (design wavelength range $325-1,100 \mathrm{~nm}$ ) and an uncoated Glan-Laser calcite polarizer were added to the experimental set-up.

In all experiments, hard-coated bandpass filters with $10-\mathrm{nm}$ full-width at half-maximum of their transmission peak were placed in front of the detector to isolate scattered light within the desired wavelength range. The signal from the PMT was pre-amplified five-times before entering a photon counter. The photon counter was used in the gated regime-the signal was measured for an equal period of time with the laser beam blocked and unblocked by the optical chopper to obtain an estimate of the noise and ambient counts.

\section{Data availability}

All data supporting this study are openly available from the University of Bath Research Data Archive at https://doi.org/10.15125/BATH-01005 (ref. ${ }^{58}$ ).

\section{References}

58. Ohnoutek, L., Olohan, B. J. \& Valev, V. K. Dataset for Third Harmonic Hyper Mie Scattering Optical Activity From Semiconductor Helices (University of Bath Research Data Archive, 2021); https://doi.org/10.15125/BATH-01005

59. Gaponik, N. et al. Thiol-Capping of CdTe Nanocrystals: an alternative to organometallic synthetic routes. J. Phys. Chem. B 106, 7177-7185 (2002).

60. Feng, W. et al. Assembly of mesoscale helices with near-unity enantiomeric excess and light-matter interactions for chiral semiconductors. Sci. Adv. 3, e1601159 (2017)

\section{Acknowledgements}

We are grateful to D. Carbery for providing cuvettes with $1 \mathrm{~mm}$ pathlength. V.K.V. acknowledges support from the Royal Society through the University Research Fellowships, the Royal Society grants PEF1\170015, RF\ERE\210172, ICA $\backslash R 1 \backslash 201088$ and RGF\EA 180228 , the STFC grant ST/R005842/1 and the EPSRC grant EP/ T001046/1. L.O. and V.K.V. acknowledge funding and support from the Engineering and Physical Sciences Research Council (EPSRC) Centre for Doctoral Training in Condensed Matter Physics (CDT-CMP), grant EP/L015544/1. N.A.K acknowledges the Vannevar Bush DoD Fellowship to N.A.K. titled Engineered Chiral Ceramics, ONR N000141812876, and ONR COVID-19 Newton Award Pathways to Complexity with Imperfect Nanoparticles, HQ00342010033. D.M.R. and G.D.P. acknowledge support from the EPSRC through grant EPSRC DTP EB- BB1250.

\section{Author contributions}

V.K.V. and N.A.K. conceived the experiments. L.O., V.K.V. and B.O. performed the nonlinear optical experiments. J-Y.K., J.L. and N.A.K. prepared and provided the samples. L.O., D.M.R. and G.D.P. performed the linear optical experiments. J-Y.K. performed the numerical simulations. L.O., J-Y.K., N.A.K. and V.K.V. analysed the data. L.O. and V.K.V. wrote the first draft and all authors contributed towards writing the paper.

\section{Competing interests}

The authors declare no competing interests.

\section{Additional information}

Supplementary information The online version contains supplementary material available at https://doi.org/10.1038/s41566-021-00916-6.

Correspondence and requests for materials should be addressed to

Nicholas A. Kotov or Ventsislav K. Valev.

Peer review information Nature Photonics thanks Costantino De Angelis and the other, anonymous, reviewer(s) for their contribution to the peer review of this work.

Reprints and permissions information is available at www.nature.com/reprints. 